\title{
Resveratrol protects against oxidized low-density lipoprotein-induced human umbilical vein endothelial cell apoptosis via inhibition of mitochondrial-derived oxidative stress
}

\author{
YUJIE LIU ${ }^{1}$, XIZHOU CHEN ${ }^{2}$ and JIE LI $^{3}$ \\ ${ }^{1}$ Department of Cardiology, Tongchuan Kuangwuju Central Hospital, Shaanxi Coal and Chemical Industry Group, Tongchuan, \\ Shaanxi 727000; ${ }^{2}$ Department of General Medicine, Baoji Central Hospital, Baoji, Shaanxi 721008; \\ ${ }^{3}$ Department of Anesthesiology, Sun Yat-sen Memorial Hospital, Sun Yat-sen University, \\ Guangzhou, Guangdong 510120, P.R. China
}

Received January 8, 2016; Accepted January 3, 2017

DOI: $10.3892 / \mathrm{mmr} .2017 .6304$

\begin{abstract}
Resveratrol, a natural phytochemical found in grapes and red wine, has been found to possess protective effects against endothelial cell apoptosis and oxidative damage. Oxidized-low density lipoprotein (ox-LDL) can induce apoptosis of endothelial cells, which is an important initial event in several cardiovascular diseases. However, the effect of resveratrol on ox-LDL-induced apoptosis and oxidative damage, and the possible associated mechanisms remain to be elucidated. In the present study, following exposure to ox-LDL, human umbilical vein endothelial cells (HUVECs) were treated with or without resveratrol. Cell viability was examined using Cell Counting Kit-8 and 5-bromo-2'-deoxyuridine uptake assays, respectively. Cell apoptosis was determined by flow cytometry. Apoptosis-associated markers were detected using western blot analysis. Oxidative stress was analyzed using molecular and biochemical approaches. Resveratrol restored ox-LDL-induced HUVEC injury and apoptosis in a dose-dependent manner. In addition, compared with the control group, ox-LDL treatment decreased the B cell lymphoma-2 (Bcl-2)/Bcl-2-associated X protein ratio, mitochondrial membrane potential and activation of superoxide dismutase, and enhanced the release of mitochondrial cytochrome $c$ into the cytoplasm, the activation of caspase and lipid peroxidation. All these alterations were significantly inhibited following treatment with resveratrol. The results demonstrated that resveratrol prevented HUVEC apoptosis through inhibiting mitochondria-derived oxidative damage. These findings may provide a novel mechanism by which resveratrol prevents against endothelial cell apoptosis.
\end{abstract}

Correspondence to: Professor Jie Li, Department of Anesthesiology, Sun Yat-sen Memorial Hospital, Sun Yat-sen University, 107 Yan Jiang West Road, Guangzhou, Guangdong 510120, P.R. China E-mail: 1ijie_sysu@126.com

Key words: resveratrol, endothelial cells, apoptosis, mitochondrial pathway, oxidative damage

\section{Introduction}

Atherosclerosis (AS) is the primary factor underlying cardiovascular and cerebrovascular diseases with high morbidity and mortality rates (1). Endothelial dysfunction, which is closely associated with vascular endothelial cell injury, is implicated in a number of pathophysiologic processes, including atheromatous plaque formation, coronary artery diseases, diabetes and hypertension (2-4). Of note, vascular endothelial cell injury as a result of apoptosis is essential in the initiation of AS (5).

The excessive generation of reactive oxygen species (ROS) from oxidative stress is also considered to be crucial in the development of AS, which results in apoptosis or necrosis through the induction of mitochondrial membrane depolarization and caspase signaling cascades in endothelial cells $(6,7)$. Oxidized low-density lipoprotein (ox-LDL) is the primary stimuli of oxidative stress, which accelerates smooth muscle cell proliferation, foam cell formation and endothelial cell apoptosis (8). These pathological alterations contribute to disruption of the endothelium barrier and subsequently facilitate the formation of atherosclerotic plaques (9). The increased apoptosis of endothelial cells induced by ox-LDL has been suggested to be associated with the binding of lectin-like ox-LDL receptor 1 (LOX-1) (10), activation of the caspase signaling pathway, the imbalance of B cell lymphoma-2 (Bcl-2) and $\mathrm{Bcl}-2$-associated $\mathrm{X}$ protein (Bax) (11) and the translocation of nuclear factor- $\mathrm{kB}$ (12).

Dietary polyphenols in human food are the most abundant natural antioxidants, and have been shown to be efficient in treating various diseases, including cancer and cardiovascular diseases (13). Resveratrol (trans-3,4,5-trihydroxystilbene) is a natural phytochemical found in grapes, red wine and certain Chinese medicinal herbs (14). It initially generated considerable attention due to its anticancer properties. In addition to cancer therapy, resveratrol possesses critical cardioprotective effects (15). These biological activities may be associated with its anti-inflammatory, anti-oxidative and immune-modulatory properties (16). Although increasing evidence supports that resveratrol exerts a protective effect against endothelial cell 
apoptosis and endothelial dysfunction through biological processes, including the suppression of superoxide production and regulation of the Lox-1-dependent signaling pathway (17-19), the importance of resveratrol in endothelial cell apoptosis has received less attention. Therefore, the aim of the present study was to examine the role of resveratrol in HUVEC apoptosis induced by ox-LDL and further examine its possible mechanisms.

\section{Materials and methods}

Materials. Resveratrol was purchased from Sigma-Aldrich; Merck Millipore (Darmstadt, Germany). The powder was dissolved in dimethyl sulfoxide (DMSO; $<0.1 \% \mathrm{v} / \mathrm{v}$ ) as a stock solution with a concentration of $200 \mathrm{mg} / \mathrm{ml}$. HUVECs were obtained from KeyGen Biotech Co., Ltd. (Nanjing, China). M199 cell culture medium, fetal bovine serum (FBS), endothelial growth factors (EGFs), penicillin and streptomycin were purchased from Gibco; Thermo Fisher Scientific, Inc. (Waltham, MA, USA). Ox-LDL was obtained from Yiyuan Biotechnologies (Guangzhou, China). 5,5',6,6'-tetrachloro-1,1', 3,3'-tetraethyl-benza-midazolocarbocyanin iodide (JC-1) dye, the Cell Counting Kit-8 (CCK-8) assay and 2',7'-dichlorodihydrofluorescein diacetate (DCFH-DA) were obtained from Beyotime Institute of Biotechnology (Jiangsu, China). Antibodies targeting 5-bromo-2'-deoxyuridine (BrdU; cat. no. sc-70443; 1:2,000), Bcl-2 (cat no. sc-509; $1: 2,000)$, Bax (cat. no. sc-70407; 1:2,000), cytochrome $c$ (cat. no. sc-514435; 1:2,500), caspase-3 (cat. no. sc-271759; $1: 2,500$ ), caspase-9 (cat. no. sc-81589; 1:2,500), caspase- 8 (cat. no. sc-6136; 1:2,500), poly (ADP-ribose) polymerase 1 (PARP; cat. no. sc-136208; 1:2,500), cyclooxygenase IV (COX IV) (cat. no. sc-69359; 1:2,500) and $\beta$-actin (cat. no. sc-130301; 1:4,000) were purchased from Santa Cruz Biotechnology, Inc. (Dallas, TX, USA). All other reagents were purchased from Sigma-Aldrich; Merck Millipore, unless otherwise specified.

Cell culture and treatment. The HUVECs were cultured in M199 medium supplemented with 10\% FBS, $100 \mu \mathrm{g} / \mathrm{ml} \mathrm{EGF,}$ $100 \mathrm{U} / \mathrm{ml}$ penicillin and $100 \mu \mathrm{g} / \mathrm{ml}$ streptomycin at $37^{\circ} \mathrm{C}$ in a humidified atmosphere of $95 \%$ air; $5 \% \mathrm{CO}_{2}$. In selected experiments, the HUVECs were incubated with various concentrations of ox-LDL (30, 60,90, 120, 150 and $180 \mu \mathrm{g} / \mathrm{ml})$, with or without resveratrol $(5,10,20,40,80$ and $120 \mu \mathrm{g} / \mathrm{ml})$, for $24 \mathrm{~h}$ at $37^{\circ} \mathrm{C}$.

Cell viability. Cell viability was measured using CCK-8 and BrdU assays, according to an established method (20). The HUVECs were seeded in 96-well plates at a density of $2 \times 10^{5}$ cells/well and then exposed to different treatments, as indicated. Finally, CCK-8 (10 $\mu \mathrm{l} /$ well) was added and incubated at $37^{\circ} \mathrm{C}$ for $2 \mathrm{~h}$. The absorbance was read at $450 \mathrm{~nm}$ using a plate reader (BioTek Instruments, Inc., Winooski, VT, USA). The BrdU assay was measured according to BrdU uptake. The cells were incubated with $50 \mathrm{mM}$ BrdU for $4 \mathrm{~h}$ at $37^{\circ} \mathrm{C}$ and then fixed with $4 \%$ paraformaldehyde. Following being permeabilized with $0.1 \%$ Triton $\mathrm{X}-100$ for $5 \mathrm{~min}$, the samples were incubated with anti-BrdU monoclonal antibody at $4^{\circ} \mathrm{C}$ overnight, followed by treatment with biotinylated goat anti-mouse IgG antibody (Santa Cruz Biotechnology, Inc.; cat. no. sc-130301; 1:4,000) for $1 \mathrm{~h}$ at room temperature. The percentage of $\mathrm{BrdU}^{+}$cells was determined by counting the numbers of stained cells and total cells using a light microscope (Olympus Corporation, Tokyo, Japan).

Analysis of apoptosis. HUVEC apoptosis was measured using an Annexin V-FITC Apoptosis Detection kit (KeyGEN Biotech Co., Ltd.) using flow cytometry according to the manufacturer's protocol. Briefly, the HUVECs were trypsinized and then harvested by centrifugation at 12,000 $\mathrm{x} g$ at $4^{\circ} \mathrm{C}$ for $15 \mathrm{~min}$. The cells were suspended in a binding buffer of Annexin V-FITC and propidium iodide (PI) at room temperature in the dark for $15 \mathrm{~min}$. The stained samples were then analyzed using flow cytometry (Beckman Coulter, Inc., Fullerton, CA, USA) and the percentage of apoptotic cells was reflected by the Annexin V/PI ratio. Annexin V was set as the horizontal axis and PI as the vertical axis.

Western blot analysis. The HUVECs were collected and lysed in RIPA lysis buffer (Cell Signaling Technology, Inc., Danvers, MA, USA) containing protease and phosphatase inhibitor cocktail. The protein concentration was determined using a BCA protein content kit (Beyotime Institute of Biotechnology). Proteins $(40 \mu \mathrm{g})$ were separated using $10 \%$ sodium dodecyl sulfate polyacrylamide gel electrophoresis (SDS-PAGE) and electrotransferred onto PVDF membranes (EMD Millipore, Billerica, MA, USA). The membranes were blocked with 5\% non-fat milk for $1 \mathrm{~h}$ at room temperature and then incubated with the following appropriate primary antibodies overnight at $4^{\circ} \mathrm{C}$ : Bcl-2, Bax; cytochrome $c, \mathrm{COX}$ IV, cleaved caspase-9, cleaved caspase-3, cleaved caspase-8, cleaved-PARP and $\beta$-actin. Following incubation with the secondary antibodies including horseradish peroxidase-conjugated rabbit anti-mouse (cat. no. 58802; 1:1,000; Cell Signaling Technology, Inc.) or horseradish peroxidase-conjugated donkey anti-goat (cat. no. sc-2056; 1:1,000; Santa Cruz Biotechnology, Inc.) for $1 \mathrm{~h}$ at room temperature, the signals were determined using an ECL kit and the intensity of the protein bands was analyzed using Image J software (version 1.41; National Institutes of Health, Bethesda, MA, USA).

Isolation of mitochondria. Intact mitochondria were isolated using a Mitochondria Isolation kit (Thermo Fisher Scientific, Inc.) according to the manufacturer's protocol. The cytosolic and mitochondrial fractions were used for western blot analysis. Cox IV was used as the loading control for the mitochondrial fraction.

Mitochondria membrane potential (MMP) analysis. MMP was measured using JC-1, which is an indicator of the loss of MMP. The aggregate red fluorescent form indicates healthy MMP, whereas the green fluorescent monomeric form indicates loss of MMP. The fluorescence was observed using a confocal laser scanning microscope (Zeiss 710; Zeiss GmbH, Munich, Germany) and the fluorescence intensity was calculated using ImageJ software (NIH).

ROS detection. ROS in the HUVECs was visualized using 2',7'-dichlorodihydrofluorescein diacetate (DCFH-DA) as 
A

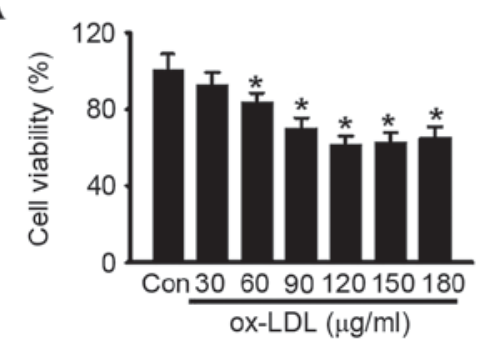

D

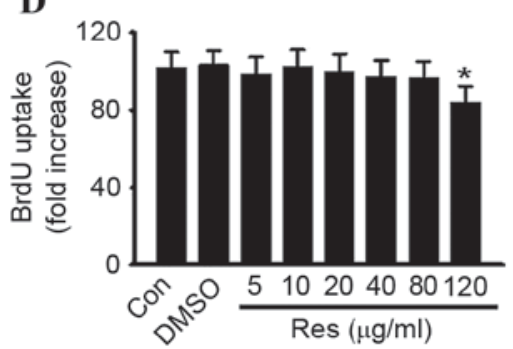

B

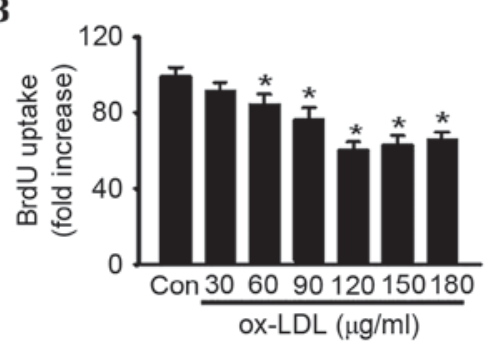

E

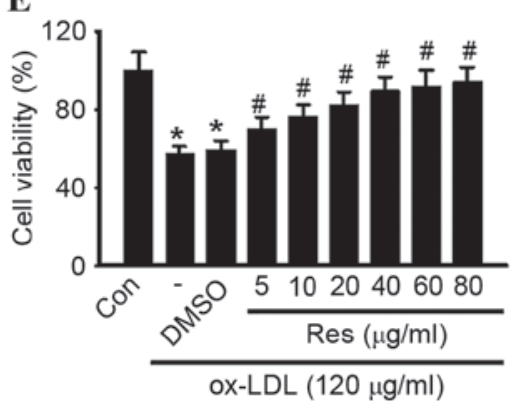

C

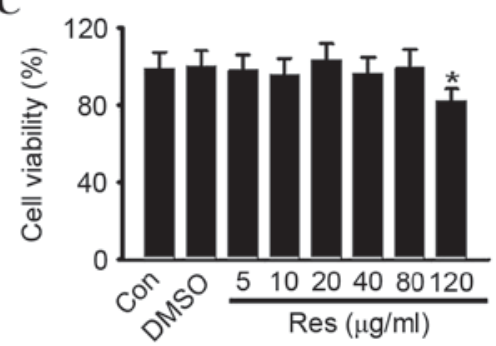

$\mathbf{F}$

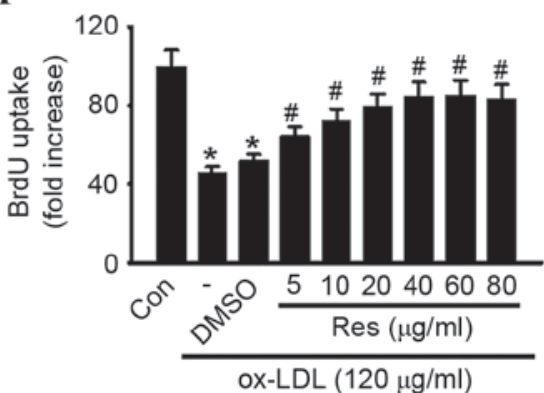

Figure 1. Res treatment inhibits ox-LDL-induced HUVEC injury. (A and B) Cell viability of HUVECs treated with various concentrations of ox-LDL for $24 \mathrm{~h}$ was evaluated using (A) CCK-8 and (B) BrdU assays. HUVECs were treated with different concentrations of Res for 24 h. Cell viability was examined using (C) CCK-8 and (D) BrdU assays. Cell viability was examined using (E) CCK-8 and (F) BrdU assays. The decrease in HUVEC cell viability induced by ox-LDL was restored by Res in a dose-dependent manner. All data are presented as the mean + standard error of the mean. ${ }^{*} \mathrm{P}<0.05$, vs. control; ${ }^{\#} \mathrm{P}<0.05$, vs. ox-LDL group alone $(\mathrm{n}=6)$. HUVECs, human umbilical vein endothelial cells; ox-LDL, oxidized-low density lipoprotein; Res, resveratrol; Con, control; CCK-8, Cell Counting Kit-8; BrdU, 5-bromo-2'-deoxyuridine.

previously described (21). Briefly, the HUVECs were washed with PBS three times and incubated with DCFH-DA $(5 \mu \mathrm{M})$ in serum-free M199 medium for $30 \mathrm{~min}$ at $37^{\circ} \mathrm{C}$ in the dark. The cells were then washed in PBS and images were captured using a fluorescence microscope (BX61; Olympus Corporation, Tokyo, Japan) with an excitation wavelength of $488 \mathrm{~nm}$ and emission wavelength of $525 \mathrm{~nm}$. The fluorescence intensity was calculated using ImageJ software (NIH).

Superoxide dismutase (SOD) and lipid peroxidation assay. The activity of SOD was measured using a commercial kit (Beyotime Institute of Biotechnology) according to the manufacturer's protocol. Additionally, the level of lipid peroxidation was detected using a malondialdehyde (MDA) assay kit (Beyotime Institute of Biotechnology). The activity of SOD and levels of MDA are presented as U/mg protein and $\mathrm{nmol} / \mathrm{mg}$ protein, respectively.

Statistical analysis. All data are expressed as the mean \pm standard error of the mean from at least 4 independent experiments. Data analysis was performed using one-way or two-way analysis of variance using SPSS 16.0 software (SPSS, Inc., Chicago, IL, USA). $\mathrm{P}<0.05$ was considered to indicate a statistically significant difference.

\section{Results}

Resveratrol reverses ox-LDL-induced apoptosis in HUVECs. To confirm the pro-apoptotic effect of ox-LDL in HUVECs, the cells were treated with various concentrations of ox-LDL $(30,60,90,120,150$ and $180 \mu \mathrm{g} / \mathrm{ml})$ for $24 \mathrm{~h}$, and cell viability was examined using CCK- 8 and BrdU assays, respectively.
Compared with the control group, the cell viability of the HUVECs was significantly decreased, in a dose-dependent manner, in the cells treated with ox-LDL (Fig. 1A). At concentrations of $30,60,90,120,150$ and $180 \mu \mathrm{g} / \mathrm{ml}$, cell viability decreased from $100.5 \pm 8.61 \%$ to $92.6 \pm 6.7,83.9 \pm 4.8,70.3 \pm 5.2$, $61.5 \pm 4.1,62.8 \pm 4.9$ and $64.8 \pm 5.8 \%$, respectively. The maximal inhibitory effect of ox-LDL on cell viability was observed at a concentration of $120 \mu \mathrm{g} / \mathrm{ml}$. Similar results were obtained using a BrdU uptake assay (Fig. 1B). A concentration of $120 \mu \mathrm{g} / \mathrm{ml}$ ox-LDL was selected to perform the experiments. The CCK-8 and BrdU assays showed that resveratrol alone had no cytotoxic effect towards the HUVECs at concentrations $<120 \mu \mathrm{g} / \mathrm{ml}$ (Fig. 1C and D). To investigate whether resveratrol protected against ox-LDL-induced cell injury, the HUVECs were treated with ox-LDL $(120 \mu \mathrm{g} / \mathrm{ml})$ for $24 \mathrm{~h}$ in the presence or absence of resveratrol, and cell viability was examined. As shown in Fig. 1E, resveratrol attenuated ox-LDL induced cell injury dose-dependently. In accordance with the results of the CCK-8 assay, a similar trend was observed in the BrdU uptake assay (Fig. 1F). Notably, no significant difference was found between 40 and $80 \mu \mathrm{g} / \mathrm{ml}$. Thus, $40 \mu \mathrm{g} / \mathrm{ml}$ resveratrol was selected for use in the following experiments. Using Annexin V-FITC/PI staining, the apoptotic rates of the cells treated with ox-LDL in the presence or absence of resveratrol were quantified by flow cytometry (Fig. 2A and B). Compared with the control, the percentage of apoptotic cells was significantly increased in the HUVECs treated with ox-LDL. However, the increased apoptotic rate was significantly inhibited following resveratrol exposure.

Effect of resveratrol on ox- $L D L$-induced expression of $B c l-2$ and Bax in HUVECs. To investigate the mechanism by 
A

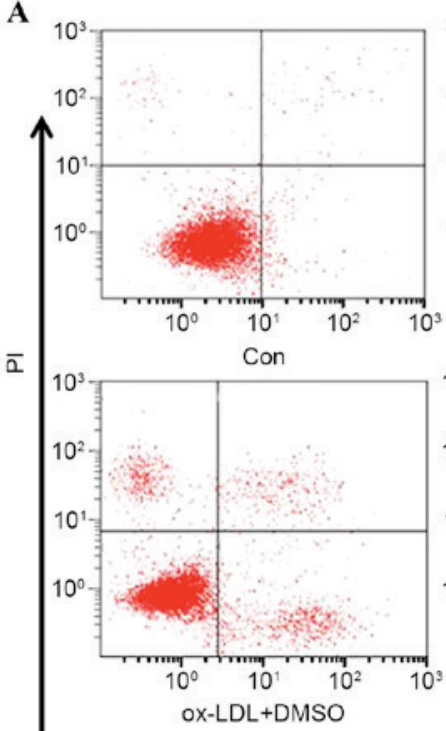

Annexin V-FITC

D

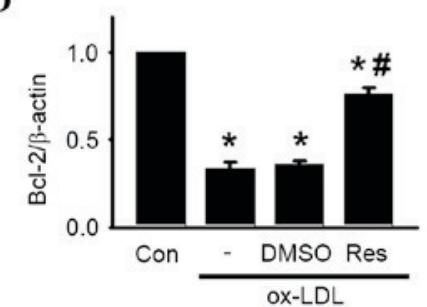

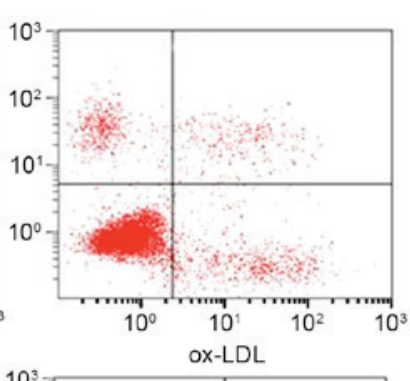

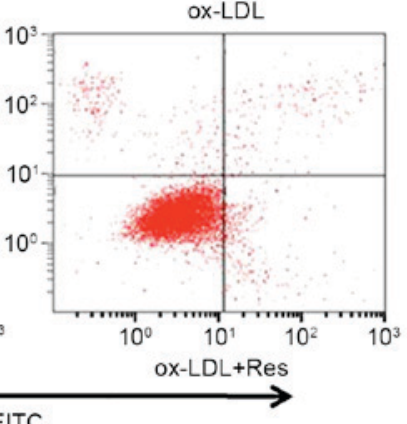

E

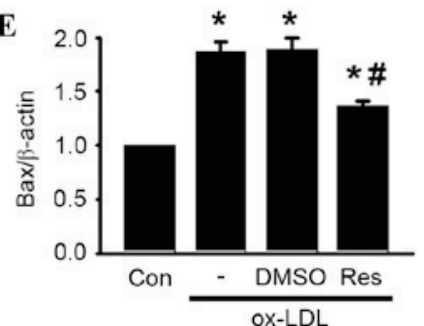

C
B
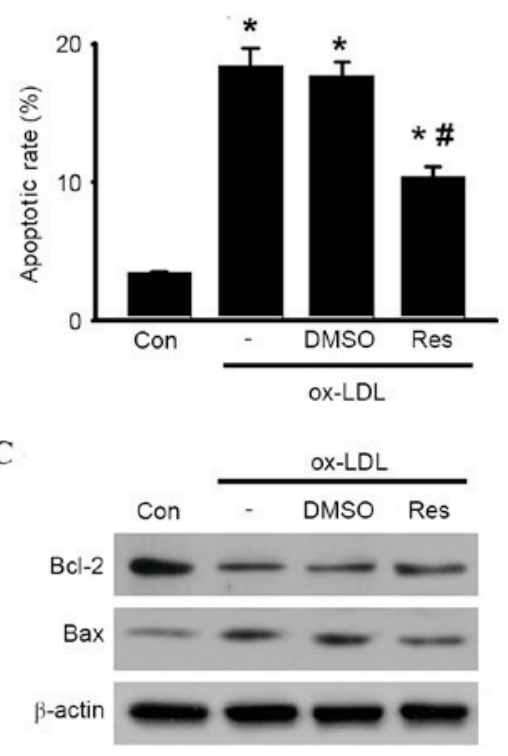

F

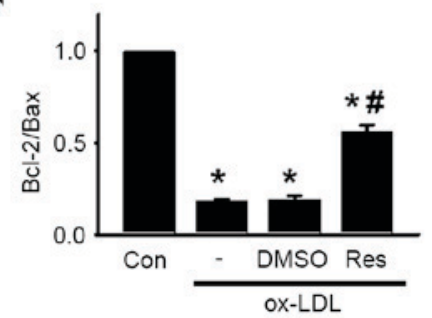

Figure 2. Res attenuates ox-LDL-induced apoptosis of HUVECs. Cells were incubated with ox-LDL $(120 \mu \mathrm{g} / \mathrm{ml})$ in the presence or absence of Res $(40 \mu \mathrm{g} / \mathrm{ml})$ for 24 h. (A) Cell apoptosis was examined using Annexin V/PI staining followed by flow cytometry. (B) Percentages of apoptotic cells were calculated. (C) Protein expression levels of Bcl-2 and Bax were measured using western blot analysis. Representative western blot images are shown. (D) Densitometric analysis of Bcl-2, (E) Bax and (F) Bcl-2/Bax ratio. All data are presented as the mean + standard error of the mean. ${ }^{\text {P }}<<0.05$, vs. control; ${ }^{\text {"P }}<0.05$, vs. ox-LDL alone $(n=4)$. HUVECs, human umbilical vein endothelial cells; ox-LDL, oxidized-low density lipoprotein; Res, resveratrol; Con, control; PI, propidium iodide; Bcl-2, B cell lymphoma-2; Bax, Bcl-2-associated X protein; DMSO, dimethyl sulfoxide.

which resveratrol attenuated ox-LDL-induced apoptosis in HUVECs, the protein expression levels of Bcl-2 and Bax were determined. Bcl-2 is an anti-apoptotic protein, which inhibits programmed cell death; Bax is a pro-apoptotic protein, which drives the cell towards apoptosis (22). The ratio of Bcl-2/Bax appears to be a determinant of the survival or death of cells. As shown in Fig. 2C-F, ox-LDL treatment decreased the protein expression of $\mathrm{Bcl}-2$ and increased the protein expression of Bax, resulting in a decrease in the Bcl-2/Bax ratio. By contrast, resveratrol attenuated the ox-LDL-induced decrease of the Bcl-2/Bax ratio, which was due to the increased level of Bcl-2 and decreased level of Bax.

Effects of resveratrol on the ox-LDL-induced alteration of $M M P$. The loss of MMP is a distinguishing feature during the process of cell apoptosis (23). To investigate the association between resveratrol and mitochondrial membrane depolarization in HUVECs, the present study examined the MMP using JC-1 staining. Following ox-LDL treatment, the red fluorescence of JC-1 was significantly decreased and the green fluorescence was markedly increased, resulting in an augmented ratio of green/red fluorescence intensity (Fig. 3A and B). As expected, the increase in the green/red fluorescence ratio induced by ox-LDL was counteracted by resveratrol treatment. These results suggested that resveratrol inhibited ox-LDL-induced apoptosis via the stabilization of MMP.

Resveratrol inhibits HUVEC apoptosis induced by ox-LDL through the mitochondria-dependent pathway. It has been documented that MMP destabilization leads to the release of cytochrome $c$, sequentially initiating activation of the caspase cascade, including caspase-3 and caspase-9 (24). The results of the western blot analysis showed that treatment with ox-LDL enhanced the release of cytochrome $c$ from the mitochondria to the cytosol. The increased release of cytochrome $c$ induced by ox-LDL was alleviated by resveratrol exposure (Fig. 3C-F). Subsequently, the levesl of caspase-9, caspase-3, PARP and caspase- 8 cleavage was determined in the HUVECs. The western blot analysis revealed that the cleavage of caspase-9, caspase-3 and PARP were significantly increased following ox-LDL treatment. However, these elevations were inhibited by resveratrol treatment (Fig. 4A-C). Of note, the level of caspase- 8 cleavage remained unaltered in the presence or absence of resveratrol (Fig. 4D) Taken together, these results indicated that resveratrol inhibited ox-LDL-induced apoptosis through the mitochondria-dependent pathway instead of the extrinsic death receptor-mediated pathway. 
A

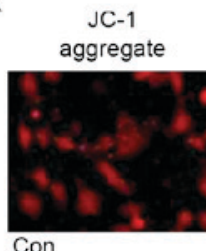

Con

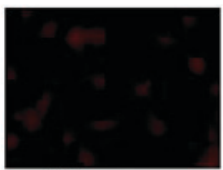

ox-LDL

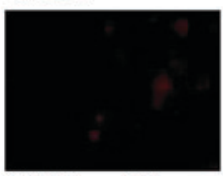

DMSO+ox-LDL

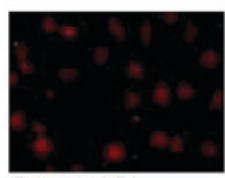

Res+ox-LDL
JC-1 monomer
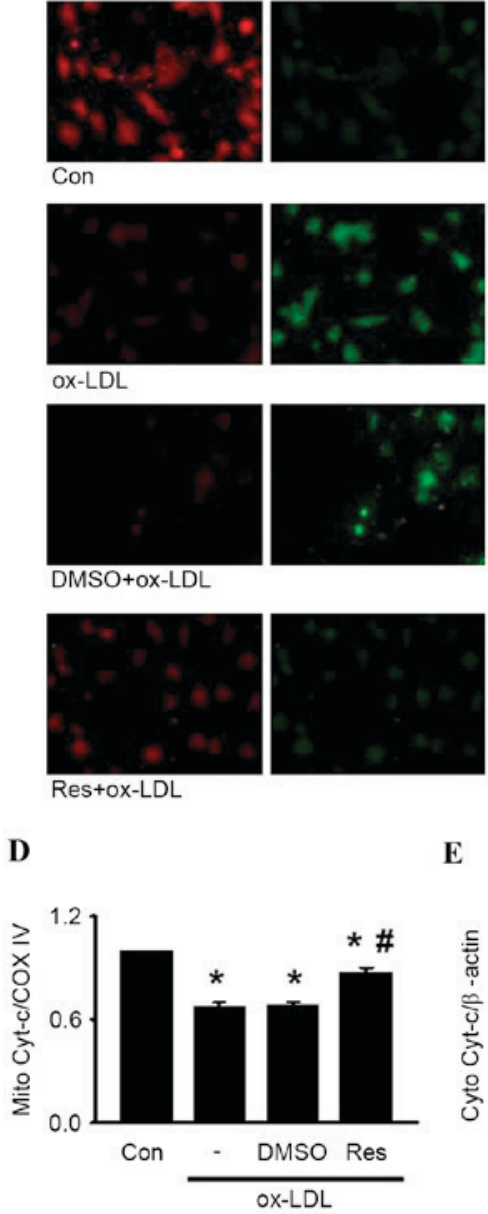

E

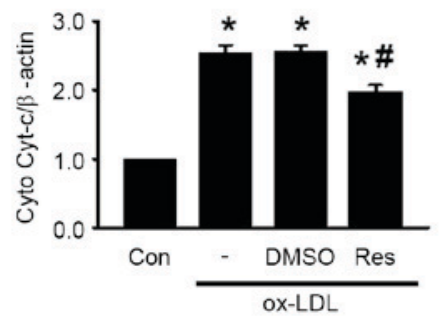

B

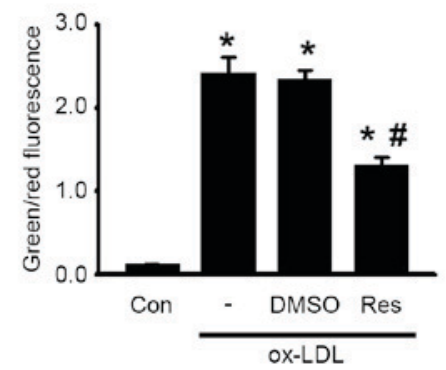

C

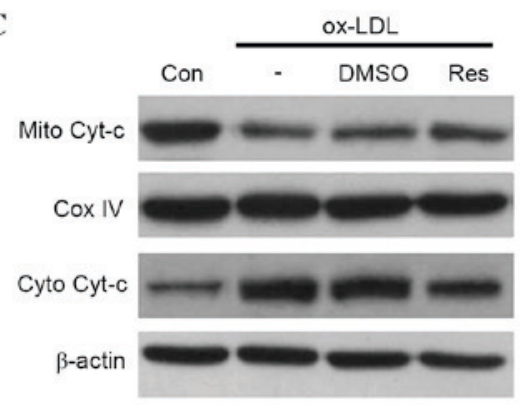

F

Figure 3. Res inhibits ox-LDL-induced apoptosis in HUVECs through the mitochondria-dependent pathway. HUVECs were treated with ox-LDL (120 $\mu$ g/ml) in the presence or absence of Res $(40 \mu \mathrm{g} / \mathrm{ml})$ for $24 \mathrm{~h}$. (A) Mitochondrial membrane potential was measured using JC-1 dye staining under confocal microscopy. Green fluorescence represents JC-1 monomer and red fluorescence represents JC-1 aggregate; merged images show combined green and red images (x400). (B) Quantitative analysis of the ratio of green/red fluorescence (C) Protein expression of cytochrome $c$ in mitochondria and cytosol were measured using western blot analysis. Densitometric analyses of the expression of cytochrome $c$ in the (D) mitochondria and (E) cytosol. (F) Densitometric analysis of the release of cytochrome $c$ from the mitochondria to the cytoplasm. All data are presented as the mean + standard error of the mean. ${ }^{*} \mathrm{P}<0.05$, vs. control; ${ }^{\#} \mathrm{P}<0.05 \mathrm{~m}$ vs. ox-LDL group (n=4-6). HUVECs, human umbilical vein endothelial cells; ox-LDL, oxidized-low density lipoprotein; Res, resveratrol; Con, control; DMSO, dimethyl sulfoxide; JC-1, 5,5', 6,6'-tetrachloro-1,1', 3,3'-tetraethyl-benza-midazolocarbocyanin iodide; Mito, mitochondrial; Cyto, cytosolic; Cyt-c, cytochrome $c$; COX IV, cyclooxygenase IV.

Resveratrol protects HUVECs from oxidative damage induced by $o x-L D L$. Oxidative damage can be caused by excessive intracellular ROS generation, a critical contributor to the pathological process of endothelial cell apoptosis and atherosclerosis $(7,10)$. To elucidate the effects of resveratrol on the oxidative stress induced by ox-LDL in HUVECs, the present study examined ROS generation using DCFH-DA. As shown in Fig. 5A and B, DCF fluorescence intensity was significantly increased in the presence of ox-LDL, which was alleviated by resveratrol. Similarly, resveratrol decreased ox-LDL-induced lipid peroxidation, indicated by MDA generation (Fig. 5C). By contrast, the activity of SOD, an antioxidant enzyme, was markedly decreased by ox-LDL. As expected, resveratrol markedly attenuated the decreased activity of SOD (Fig. 5D). Together, these data suggested that resveratrol inhibited ox-LDL-induced oxidative damage, which may be associated with its protective effect on endothelial cell apoptosis.

\section{Discussion}

Epidemiological studies have revealed a negative association between cardiovascular disease-associated mortality and the consumption of red wine $(25,26)$. This has been documented as the 'French paradox', which partly indicates that the resveratrol present in red wine may assist in preventing cardiovascular diseases (27). A number of molecular targets have been found to be modulated by resveratrol in several cardiovascular diseases, including atherosclerosis, hypertension and inflammation $(28,29)$. Although resveratrol has been shown to contribute to improvements in endothelial dysfunction, its effect on endothelial apoptosis, the initial event of atherosclerosis, remains to be poorly elucidated. In the present study, it was demonstrated that resveratrol restored ox-LDL-induced endothelial cell apoptosis. The underlying mechanisms included alteration in the activation of Bcl-2/Bax proteins and associated proteins of the intrinsic 
A

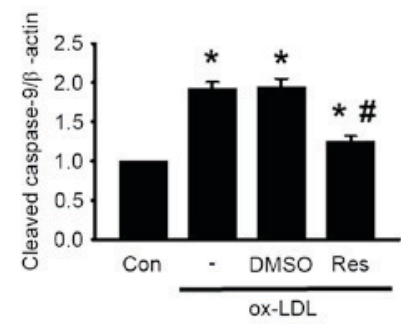

Cleaved caspase- 9

$\beta$-actin

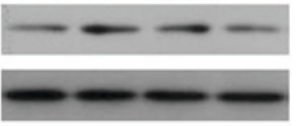

C

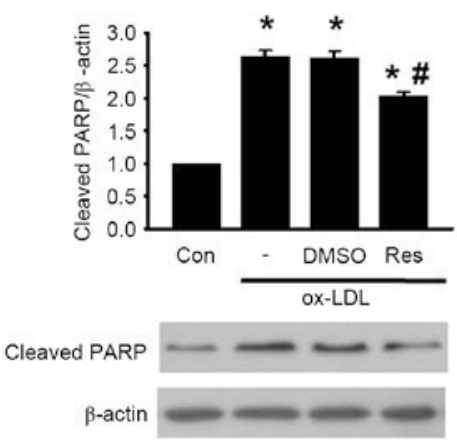

B
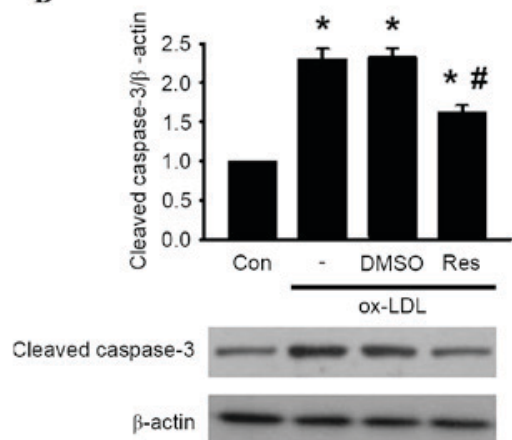

D

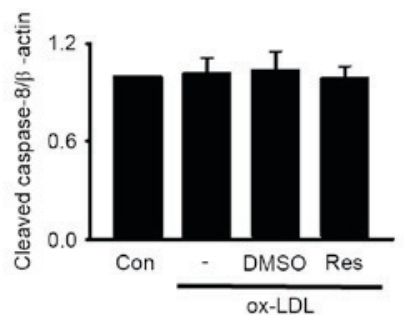

Cleaved caspase-8

$\beta$-actin

Figure 4. Res reverses ox-LDL-induced caspase activation. HUVECs were co-incubated with ox-LDL (120 $\mu \mathrm{g} / \mathrm{ml})$ and Res (40 $\mu \mathrm{g} / \mathrm{ml})$ for $24 \mathrm{~h}$. Expression levels of (A) cleaved caspase-9, (B) cleaved caspase-3, (C) cleaved PARP and (D) cleaved caspase-8 were determined using western blot analysis. Densitometric analysis showed that Res inhibited the increased expression of cleaved caspase-9, cleaved caspase- 3 and cleaved PARP, but had no effect on the expression of cleaved caspase-8. Representative western blots are shown. All data are presented as the mean + standard error of the mean. ${ }^{*} \mathrm{P}<0.05$, vs. control; ${ }^{*} \mathrm{P}<0.05$, vs. ox-LDL group ( $n=3)$. HUVECs, human umbilical vein endothelial cells; ox-LDL, oxidized-low density lipoprotein; Res, resveratrol; Con, control; DMSO, dimethyl sulfoxide; PARP, poly (ADP-ribose) polymerase 1.

A
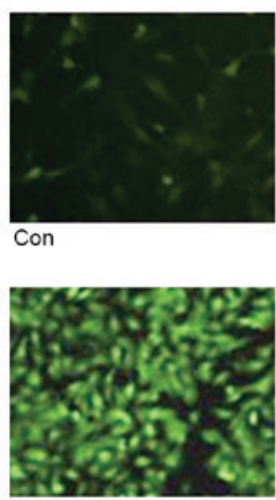

DMSO+OX-LDL

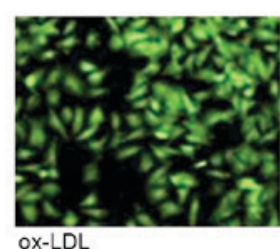

OX-LDL

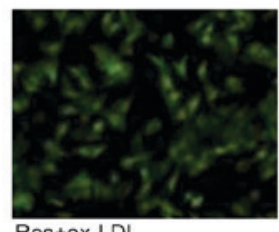

Res+ox-LDL

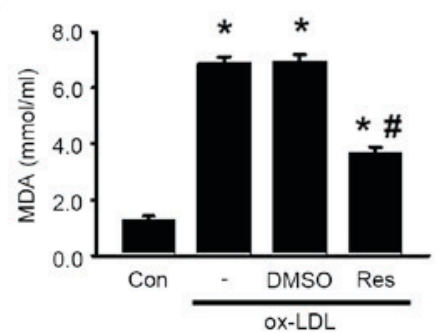

B

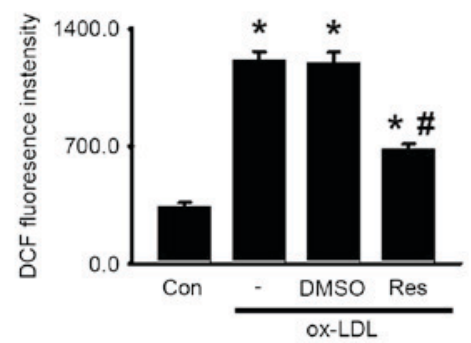

D

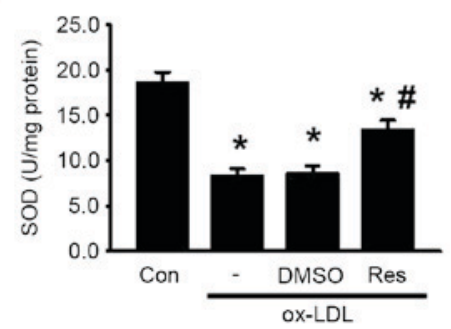

Figure 5. Res attenuates oxidase damage induced by ox-LDL in HUVECs. HUVECs were treated with ox-LDL (120 $\mu \mathrm{g} / \mathrm{ml})$ in the presence or absence of Res $(40 \mu \mathrm{g} / \mathrm{ml})$ for $24 \mathrm{~h}$. (A) ROS generation was measured. Representative images of HUVECs loaded with DCFH-DA $(5 \mu \mathrm{M})$ were captured using a fluorescence microscope (x200). (B) Quantitative analysis of DCF fluorescence intensity. (C) Levels of lipid peroxidation were detected using an MDA assay kit. (D) Intracellular activity of SOD was decreased by treatment of ox-LDL, which were inhibited by Res. Data are presented as the mean \pm standard error of the mean. "P $<0.05$, vs. control; " $\mathrm{P}<0.05$, vs. ox-LDL group $(\mathrm{n}=4)$. HUVECs, human umbilical vein endothelial cells; ox-LDL, oxidized-low density lipoprotein; Res, resveratrol; Con, control; DMSO, dimethyl sulfoxide; ROS, reactive oxygen species; DCF, 2',7'-dichlorodihydrofluorescein; MDA, malondialdehyde; SOD, superoxide dismutase. 
mitochondrial pathway in HUVECs following ox-LDL treatment. In addition, the present study found that resveratrol attenuated ox-LDL-induced oxidative damage, as evidenced by the attenuation of ROS and MDA generation, and the restoration of SOD activity.

Endothelial cell apoptosis has been implicated in the development of a variety of vascular diseases, including atherosclerosis (5). Of note, a previous study showed that increased apoptotic endothelial cells were observed in atheromatous plaques, resulting in monocyte adhesion and foam cell formation (7). Thus, the identification of effective regimens with low toxicity for the protection against endothelial cell apoptosis is urgently required. In the present study, it was found that resveratrol dose-dependently reversed ox-LDL-induced HUVEC injury and apoptosis. Of note, resveratrol had no cytotoxic effect on HUVECs at concentrations $<120 \mu \mathrm{g} / \mathrm{ml}$, indicating the safety of resveratrol for clinical use.

Abnormal expression of the Bcl-2 family is usually associated with the apoptotic process, consisting of alterations in the protein levels of anti-apoptotic Bcl-2 and pro-apoptotic Bax. Upon apoptotic stimulation, the expression of Bax is increased, leading to a lower level of Bcl-2 (30). Bax forms oligomers and transfers from the cytoplasm to the mitochondrial membrane, resulting in mitochondrial membrane depolarization (31). Subsequently, cytochrome $c$ is released from the mitochondria to the cytosol, and triggers caspase pathway activation (23). In the present study, it was demonstrated that ox-LDL resulted in an increase in the expression of Bax, and a decrease in the expression of $\mathrm{Bcl}-2$ and the $\mathrm{Bcl}-2 / \mathrm{Bax}$ ratio, all of which were attenuated following exposure to resveratrol. Furthermore, resveratrol attenuated ox-LDL-induced mitochondrial membrane depolarization and cytochrome $c$ release. Consequently, resveratrol inhibited the elevation of caspase-9, caspase-3 and PARP cleavage induced by ox-LDL, suggesting that resveratrol prevented ox-LDL-induced apoptosis in the HUVECs predominantly through the mitochondria-dependent pathway. Of note, neither ox-LDL nor resveratrol affected caspase- 8 activation, indicating that the extrinsic death receptor-mediated pathway was not involved in this process.

Oxidative damage induced by excessive ROS generation is known to be a potential inducer of apoptosis, and mitochondrial pathway dysfunction is the major cause of the overproduction of ROS $(31,32)$. Additionally, accumulating evidence has indicated that resveratrol improves cardiovascular disorders via reducing oxidative stress and damage $(33,34)$. Therefore, the present study aimed to examine whether resveratrol inhibited oxidative damage and subsequently protected HUVECs from apoptosis. The results showed that resveratrol inhibited the ox-LDL-induced increased ROS generation and lipid peroxidation, and decreased SOD activity.

In conclusion, the data obtained in the present study demonstrated the protective role of resveratrol on endothelial cell apoptosis induced by ox-LDL. Resveratrol effectively inhibited ox-LDL-induced apoptosis through inhibition of the mitochondrial pathway and oxidative damage. This novel mechanism may assist in elucidating the functional role of resveratrol for the treatment of endothelial cell apoptosis and atherosclerosis.

\section{Acknowledgements}

This study was supported by the Fundamental Research Funds for the Central Universities in China (grant no. 12ykpy26), the Guangdong Natural Science Foundation (grant nos. S2012040008068 and S2013010014514) and the Guangdong Province-Ministry of Education Joint Research Program (grant no. 2012B091100454).

\section{References}

1. Go AS, Mozaffarian D, Roger VL, Benjamin EJ, Berry JD, Blaha MJ, Dai S, Ford ES, Fox CS, Franco S, et al: Executive summary: Heart disease and stroke statistics-2014 update: A report from the American Heart Association. Circulation 129: 399-410, 2014.

2. Rajendran P, Rengarajan T, Thangavel J, Nishigaki Y, Sakthisekaran D, Sethi G and Nishigaki I: The vascular endothelium and human diseases. Int J Biol Sci 9: 1057-1069, 2013.

3. Griendling KK and FitzGerald GA: Oxidative stress and cardiovascular injury: Part I: Basic mechanisms and in vivo monitoring of ROS. Circulation 108: 1912-1916, 2003.

4. Park YS, Kim J, Misonou Y, Takamiya R, Takahashi M, Freeman MR and Taniguchi N: Acrolein induces cyclooxygenase-2 and prostaglandin production in human umbilical vein endothelial cells: Roles of p38 MAP kinase. Arterioscler Thromb Vasc Biol 27: 1319-1325, 2007.

5. Schiro A, Wilkinson FL, Weston R, Smyth JV, Serracino-Inglott F and Alexander MY: Endothelial microparticles as conveyors of information in atherosclerotic disease. Atherosclerosis 234: 295-302, 2014.

6. Fiers W, Beyaert R, Declercq W and Vandenabeele P: More than one way to die: Apoptosis, necrosis and reactive oxygen damage. Oncogene 18: 7719-7730, 1999

7. Li H, Horke S and Förstermann U: Vascular oxidative stress, nitric oxide and atherosclerosis. Atherosclerosis 237: 208-219, 2014.

8. Mitra S, Deshmukh A, Sachdeva R, Lu J and Mehta JL: Oxidized low-density lipoprotein and atherosclerosis implications in antioxidant therapy. Am J Med Sci 342: 135-142, 2011.

9. Tricot O, Mallat Z, Heymes C, Belmin J, Leseche G and Tedgui A: Relation between endothelial cell apoptosis and blood flow direction in human atherosclerotic plaques. Circulation 101: 2450-2453, 2000.

10. Lu J, Mitra S, Wang X, Khaidakov M and Mehta JL: Oxidative stress and lectin-like ox-LDL-receptor LOX-1 in atherogenesis and tumorigenesis. Antioxid Redox Signal 15: 2301-2333, 2011.

11. Chen TG, Chen TL, Chang HC, Tai YT, Cherng YG, Chang YT and Chen RM: Oxidized low-density lipoprotein induces apoptotic insults to mouse cerebral endothelial cells via a Bax-mitochondria-caspase protease pathway. Toxicol Appl Pharmacol 219: 42-53, 2007.

12. Li D and Mehta JL: Intracellular signaling of LOX-1 in endothelial cell apoptosis. Circ Res 104: 566-568, 2009.

13. Aluyen JK, Ton QN, Tran T, Yang AE, Gottlieb HB and Bellanger RA: Resveratrol: Potential as anticancer agent. J Diet Suppl 9: 45-56, 2012.

14. Cavallaro A, Ainis T, Bottari C and Fimiani V: Effect of resveratrol on some activities of isolated and in whole blood human neutrophils. Physiol Res 52: 555-562, 2003.

15. Wang H, Yang YJ, Qian HY, Zhang Q, Xu H and Li JJ: Resveratrol in cardiovascular disease: What is known from current research? Heart Fail Rev 17: 437-448, 2012.

16. Nakata R, Takahashi $\mathrm{S}$ and Inoue $\mathrm{H}$ : Recent advances in the study on resveratrol. Biol Pharm Bull 35: 273-279, 2012.

17. Li H and Förstermann U: Resveratrol: A multifunctional compound improving endothelial function. Editorial to: 'Resveratrol supplementation gender independently improves endothelial reactivity and suppresses superoxide production in healthy rats' by S. Soylemez et al. Cardiovasc Drugs Ther 23: 425-429, 2009.

18. Schmitt CA, Heiss EH and Dirsch VM: Effect of resveratrol on endothelial cell function: Molecular mechanisms. Biofactors 36: 342-349, 2010. 
19. Lin YL, Chang HC, Chen TL, Chang JH, Chiu WT, Lin JW and Chen RM: Resveratrol protects against oxidized LDL-induced breakage of the blood-brain barrier by lessening disruption of tight junctions and apoptotic insults to mouse cerebrovascular endothelial cells. J Nutr 140: 2187-2192, 2010.

20. Huang EW, Xue SJ, Li XY, Xu SW, Cheng JD, Zheng JX, Shi H, Lv GL, Li ZG, Li Y, et al: EEN regulates the proliferation and survival of multiple myeloma cells by potentiating IGF-1 secretion. Biochem Biophys Res Commun 447: 271-277, 2014.

21. Peng H, Zhong W, Zhao H, Chen L, Zhou X, Li F, Zhu W and Li G: Lack of PGC-1 $\alpha$ exacerbates high glucose-induced apoptosis in human umbilical vein endothelial cells through activation of VADC1. Int J Clin Exp Pathol 8: 4639-4650, 2015.

22. Green DR and Reed JC: Mitochondria and apoptosis. Science 281: 1309-1312, 1998

23. Tait SW and Green DR: Mitochondria and cell death: Outer membrane permeabilization and beyond. Nat Rev Mol Cell Biol 11: 621-632, 2010.

24. Deshmukh M and Johnson EM Jr: Evidence of a novel event during neuronal death: Development of competence-to-die in response to cytoplasmic cytochrome c. Neuron 21: 695-705, 1998.

25. Renaud SC, Gueguen R, Schenker J and d'Houtaud A: Alcohol and mortality in middle-aged men from eastern France. Epidemiology 9: 184-188, 1998.

26. Fukao H, Ijiri Y, Miura M, Hashimoto M, Yamashita T, Fukunaga C, Oiwa K, Kawai Y, Suwa M and Yamamoto J: Effect of trans-resveratrol on the thrombogenicity and atherogenicity in apolipoprotein E-deficient and low-density lipoprotein receptor-deficient mice. Blood Coagul Fibrinolysis 15: 441-446, 2004.
27. Yang X, Li X and Ren J: From French Paradox to cancer treatment: Anti-cancer activities and mechanisms of resveratrol. Anticancer Agents Med Chem 14: 806-825, 2014.

28. Perez-Vizcaino F, Duarte J and Andriantsitohaina R: Endothelial function and cardiovascular disease: Effects of quercetin and wine polyphenols. Free Radic Res 40: 1054-1065, 2006.

29. Rossi L, Mazzitelli S, Arciello M, Capo CR and Rotilio G: Benefits from dietary polyphenols for brain aging and Alzheimer's disease. Neurochem Res 33: 2390-2400, 2008.

30. Besbes S, Mirshahi M, Pocard M and Billard C: New dimension in therapeutic targeting of BCL-2 family proteins. Oncotarget 6 : 12862-12871, 2015.

31. Boatright KM and Salvesen GS: Mechanisms of caspase activation. Curr Opin Cell Biol 15: 725-731, 2003.

32. Johnson TM, Yu ZX, Ferrans VJ, Lowenstein RA and Finkel T: Reactive oxygen species are downstream mediators of p53-dependent apoptosis. Proc Natl Acad Sci USA 93: 11848-11852, 1996.

33. Ladurner A, Schachner D, Schueller K, Pignitter M, Heiss EH, Somoza V and Dirsch VM: Impact of trans-resveratrol-sulfates and -glucuronides on endothelial nitric oxide synthase activity, nitric oxide release and intracellular reactive oxygen species. Molecules 19: 16724-16736, 2014.

34. Li H, Xia N and Förstermann U: Cardiovascular effects and molecular targets of resveratrol. Nitric Oxide 26: 102-110, 2012. 